\title{
MULTI-MODE HOLOGRAPHIC WAVEGUIDE COUPLER
}

\author{
S.K. CASE and M.K. HAN \\ Department of Electrical Engineering. The University of Michigan, Ann Arbor, Michigan 48104, USA
}

Received 23 June 1975

A double-exposure thick-holograhic-grating waveguide coupler is described in which two waveguide modes can be excited with a single incident beam. Single-grating decouplers are also designed to selectively remove one of the coupled waves.

\section{Introduction}

Present couplers for integrated optical circuits [1] have been restricted to coupling one external wave into one guided wave. We concern ourselves in this report with a thick holographic grating coupler designed to provide simultaneous excitation of more than one guided mode when illuminated by a single external beam. Once the guided waves have been processed within the guide, we will want to selectively decouple one of the guided modes without perturbing the other. A single grating decoupler is designed for this purpose.

\section{Double-grating coupler}

We have shown earlier [2] that it is possible to double expose a thick grating such that it acts like a double beam splitter to diffract $50 \%$ of the incident energy into each of two diffracted orders. We use this

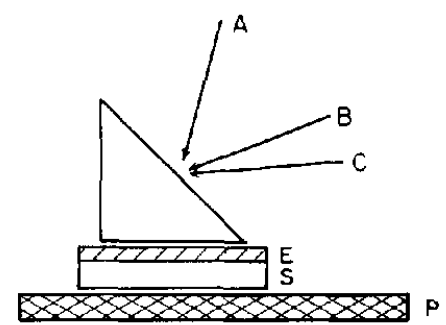

Fig. 1. Construction of a double grating coupler. A, B, C are incident plane waves; $\mathrm{E}$ is the dichromated gelatin emulsion on substrate $\mathrm{S} ; \mathrm{P}$ is an absorptive backing plate. Xylene is used as an index matching fluid between the components. principle to construct a two-mode waveguide coupler as in fig. 1. A dichromated gelatin film is exposed through a prism first with plane waves $A$ and $B$, and then with plane waves $A$ and $C$. After development, readout with common wave $A^{\prime}$ will produce both guided waves $B^{\prime}$ and $C^{\prime}$ (fig. 2) so that we can simultaneously excite two modes in the guide with a single incident beam.

We have also constructed two single-grating decouplers by exposing a piece of film through the prism with beams $\mathrm{A}$ and $\mathrm{B}$, and by exposing another piece of film with beams $A$ and $C$. Each single-grating decoupler is exposed and developed on its own $1 \mathrm{~mm}$ thick glass substrate and can now be mounted on a waveguide to couple or decouple beams of light. By choosing the construction waves $\mathrm{B}$ and $\mathrm{C}$ sufficiently far apart (in angle), we can construct the single grating decouplers such that they act independently on either of the two guided waves [3].

In fig. 3a, we show a double-grating coupler mounted on top of a $\frac{1}{4}$ " Kodak Microflat glass guide (emulsion removed) using xylene as an index matching

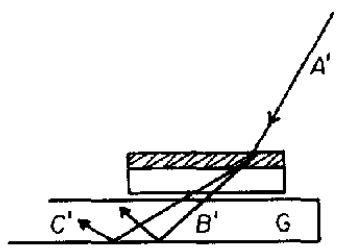

Fig. 2. Readout of the double-grating couplet with wave $A^{\prime}$ produces guided waves $B^{\prime}$ and $C^{\prime}$ in guide $G$. 


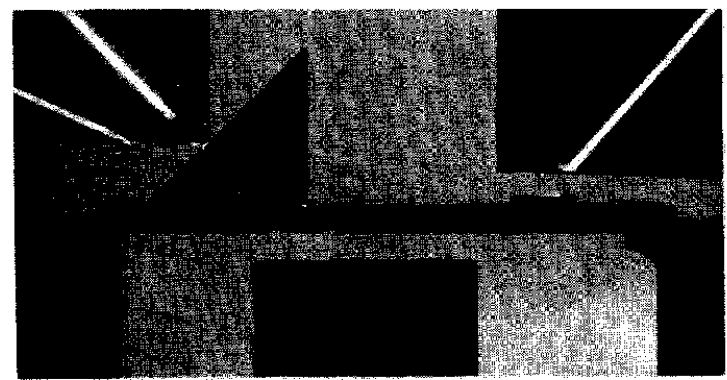

(a)

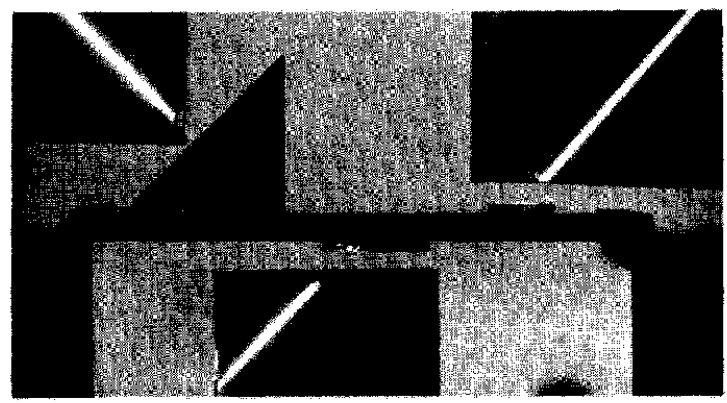

(b)

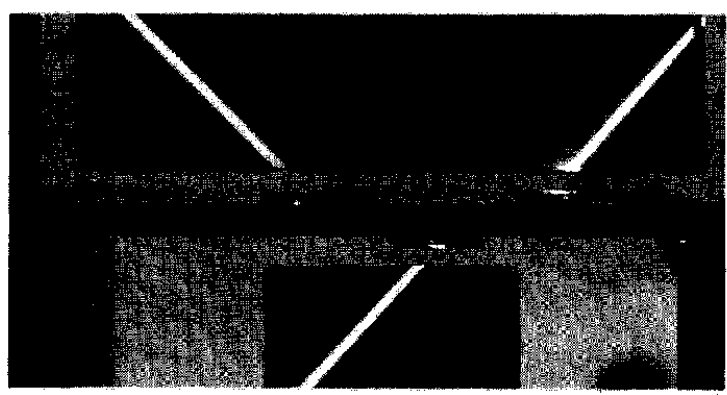

(c)

Fig. 3. Double-grating coupler: a) Two guided modes are simultaneously excited in the waveguide. b) One of the guided waves is decoupled by a single-grating decoupler. c) Both of the guided waves are decoupled by separate singlegrating decouplers.

fluid. The collimated beam of light (beam A') incident from the upper right is coupled with nearly $100 \%$ efficiency into the guided beams $\mathrm{B}^{\prime}$ and $\mathrm{C}^{\prime}$ as observed by the lack of transmitted light on the piece of ground glass below the guide. The two guided modes can be seen in this view after they have been uncoupled by a prism and illuminate a piece of ground glass held at grazing incidence. In fig. $3 \mathrm{~b}$, we attach a single-grating decoupler to the bottom of the guide. This grating was constructed with waves $\mathrm{A}$ and $\mathrm{C}$ so that on readout, it will decouple guided wave $C^{\prime}$ and diffract it into the direction of wave $A^{\prime}$. Guided beam $B^{\prime}$ is not at the Bragg angle for this decoupler and remains trapped. In fig. $3 \mathrm{c}$, we place the single grating constructed with waves A and B on top of the guide. This now decouples the energy from the remaining guided wave $\mathrm{B}^{\prime}$ into the unguided wave $\mathrm{A}^{\prime}$. The output beams can be diffracted in either an upward or downward direction by a suitable rotation of the decoupler gratings.

\section{Extensions of the principle}

This method of double-grating coupler construction (with common reference wave $A$ ) requires that the construction and readout wavelengths be the same so that the two gratings will have a common Bragg angle on readout. If the coupler is to be used at a wavelength different than that of construction, then the geometry of the construction beams must be adjusted so that the two gratings will have a common Bragg angle at the readout wavelength.

By making the mode-selective decoupler gratings sufficiently thick, their angular selectivity can be reduced so that they extract light over a very narrow angular range. In this way, the multi-mode coupler and decoupler system can be extended to include many more guided waves.

\section{Acknowledgements}

The authors wish to thank Professor Emmett Leith for helpful discussions during the course of this work. We thank the Office of Naval Research and the National Science Foundation (Grant \#GK 43148) for their generous support.

\section{References}

[1] H. Kogelnik and T.P. Sosnowski, Bell Syst. Tech. J. 49 (1970) 1602.

[2] S.K. Case, J. Opt. Soc. Am. 65 (1975) 724.

[3] R.C. Alferness and S.K. Case, J. Opt. Soc. Am. 65 (1975) 730 . 